\title{
Anticoagulants as more effective secondary prevention for embolic stroke of undetermined source: retrospective cohort study
}

\author{
Yuya Sakakura \\ Seirei Hamamatsu Byoin \\ Yoichiro Homma（ $\square$ yoh2007@sis.seirei.or.jp) \\ Seirei Hamamatsu Byoin https://orcid.org/0000-0002-3017-9078 \\ Tomoya Arakawa \\ Seirei Hamamatsu Byoin \\ Tokutaro Tanaka \\ Seirei Hamamatsu Byoin
}

Research Article

Keywords: Brain infarction, anticoagulants, antiplatelet, ESUS

Posted Date: December 13th, 2018

DOI: https://doi.org/10.21203/rs.2.88/v1

License: (c) (i) This work is licensed under a Creative Commons Attribution 4.0 International License. Read Full License 


\section{Abstract}

\section{Background:}

Whether anticoagulants or antiplatelets are more effective for secondary stroke prevention for embolic stroke of undetermined source (ESUS) remains unknown. These two treatments were compared retrospectively.

\section{Methods:}

Of 779 acute ischemic stroke inpatients between January 2012 and December 2014 in our stroke center, 110 patients met our ESUS criteria were compared. Antiplatelets were used in 81 patients, and anticoagulants were used in 29. ESUS criteria by Hart et al., except for extracranial atherosclerosis, were used. In all patients, intracranial magnetic resonance angiograms were obtained, and the infarct was diagnosed with positive diffusion studies. The primary outcome was recurrence-free survival. Score-tests for the hazard ratio assumption and for covariate effects in the Cox proportional hazard model were performed. To estimate hazard ratios, a likelihood ratio test and the Kaplan-Meier method and log-rank test were used. This study protocol was approved by the Institutional Review Board of the hospital.

\section{Results:}

The mean follow-up period was $84.0 \pm 65.5$ weeks, and 14 patients treated with antiplatelets and one treated with anticoagulants developed recurrent ischemic stroke. The antiplatelet group had a significantly higher risk of recurrence than the anticoagulant group ( $p=0.0293$, likelihood ratio $0.144,95 \%$ confidence interval [Cl]: 0.019-1.096). Multivariate analysis also showed a similar result ( $p=0.013$, likelihood ratio $-1.94,95 \%$ Cl: 0.01-0.72, respectively).

\section{Conclusions:}

These findings suggest that anticoagulants are more effective than antiplatelets for secondary stroke prevention for ESUS patients, although this was a non-randomized, single-center, retrospective analysis with a small sample size. Further studies are needed to accumulate enough evidence.

\section{Background}

Embolic stroke of undetermined source (ESUS) accounts for 10-25\% of ischemic strokes.[1, 2] It is known that ESUS is more likely to relapse than other cerebral infarctions and has a poor prognosis.[3, 4] Antiplatelet drugs are recommended for the secondary prevention of ESUS in the European and United States guidelines.[5, 6] Recent studies suggest a thrombotic mechanism for ESUS,[7] and two global studies (NAVIGATE ESUS, RE-SPECT ESUS) comparing direct oral anticoagulants (DOACs) and antiplatelets are in progress.[8, 9] In the ESUS global registry, only $7 \%$ of ESUS patients were administered anticoagulants, and the effects of anticoagulants including warfarin are still unknown.[10] On the other hand, there is no recommendation on the treatment of ESUS in Japanese guidelines.[11] It is possible that the number of ESUS patients who received anticoagulants in Japan is greater than in other countries.

\section{Objectives}

To investigate whether anticoagulants or antiplatelets are more effective for secondary stroke prevention in ESUS patients, anticoagulants were compared with antiplatelets in patients with ESUS to prevent recurrent ischemic strokes.

\section{Methods}

\section{Study design and setting}

This was a retrospective cohort study undertaken at the Stroke Center of Seirei Hamamatsu General Hospital (Hamamatsu, Japan), in which medical records between January 2012 and December 2014 were reviewed. A total of 779 ischemic stroke patients were admitted to the hospital within that period. The study was carried out in accordance with the Declaration of Helsinki, and the protocol was approved by the Institutional Review Board of Seirei Hamamatsu General Hospital. The patients were identified based on the registered disease name in the medical records, and all patients were examined to determine whether they met the ESUS criteria.

\section{ESUS criteria}

Ischemic stroke patients with a documented acute lesion on brain imaging and hospitalized at the stroke center were included. Patients who were excluded were those who did not satisfy the ESUS criteria, [1] except for extracranial atherosclerosis: (a) stroke detected by magnetic resonance imaging (MRI) that was not lacunar (defined as a subcortical infarct $\leq 2.0 \mathrm{~cm}$ on MRI diffusion images in the largest dimension, and in the distribution of the small, penetrating cerebral arteries); (b) absence of intracranial atherosclerosis causing $\geq 50 \%$ luminal stenosis in 
arteries supplying the area of ischemia; (c) no major risk of a cardioembolic source of embolism (permanent or paroxysmal atrial fibrillation, sustained atrial flutter, intracardiac thrombus, prosthetic cardiac valve, atrial myxoma or other cardiac tumors, mitral stenosis, <4 weeks after myocardial infarction, left ventricular ejection fraction $<30 \%$, valvular vegetations, or infective endocarditis); (d) no other specific cause of stroke identified (arteritis, dissection, migraine/vasospasm, drug misuse); and (e) minimum diagnostic assessment performed (brain MRI, 12-lead electrocardiogram, precordial echocardiography, cardiac monitoring for $\geq 24 \mathrm{~h}$ with automated rhythm detection, MRI or computed tomography (CT) angiography imaging of intracranial arteries supplying the area of brain ischemia and neck echo screening. Patients who were dead on discharge, those taking both anticoagulants and antiplatelets after discharge, and those taking neither anticoagulants nor antiplatelets after discharge were excluded.

\section{Patients' demographic data}

The following demographic data were collected referring to a previous cryptogenic stroke study[12] as variables: age, sex, stroke recurrence, period to recurrence, secondary prevention drug, estimated glomerular filtration rate (eGFR), CHADS $_{2}$ score,[13] modified Rankin Scale (mRS) score at discharge, death during hospitalization, and medical history of smoking, diabetes mellitus, hypertension, dyslipidemia, stroke, chronic heart failure, and acute coronary syndrome. The datasets were created by reviewing the patients' electronic medical records. The data were double-checked by two of the authors (Y.S. and T.A.).

\section{Data of the exposed group/non-exposed group}

The ESUS patients were divided into two groups: the antiplatelet (AP) group, who took antiplatelets as secondary prevention for ischemic stroke when they were discharged; and the anticoagulant (AC) group who took anticoagulants. The drug choice depended on the judgment of the doctor in charge. Antiplatelets included aspirin, clopidogrel sulfate, and cilostazol. Anticoagulants included warfarin and DOACs. DOACs were used only for deep venous thrombosis. These patients were followed for three years from ESUS onset.

\section{Outcome measures}

The primary outcome was recurrence-free survival, with recurrence defined as acute ischemic stroke including transient ischemic attacks (TIAs) evaluated by a stroke specialist regardless of positive or negative neuroimaging. The outcome measure time frame was 3 years.

Patients' overall survival and major bleeding according to the International Society on Thrombosis and Haemostasis (ISTH) criteria[14] were investigated as secondary outcomes.

\section{Statistical analysis}

The outcome variable was event-free survival time, and age, sex, $\mathrm{CHADS}_{2}$ score, and $\mathrm{mRS}$ score at discharge were used as covariates. Missing or abnormal data were checked by re-reviewing the medical charts (Y.H.). The means (standard deviation; SD) for continuous variables and frequencies (\%) for categorical variables were calculated as summary statistics.

The event-free survival rate was calculated using the Kaplan-Meier method. Crude and multivariate adjusted hazard ratios and their $95 \%$ confidence intervals were estimated by Cox proportional hazard models with the maximum likelihood method. Score test statistics were also obtained. P-values < 0.05 on two-sided tests were considered significant. SAS University (SAS Institute, Cary NC, USA) was used for statistical calculations.

The sample size was estimated based on a previous Japanese study report.[15] With a 5\% alpha error and $80 \%$ power, a sample size of 27 patients in each group was needed. A pilot study was performed with approximately 100 patients to facilitate the sample-size calculation. Consequently, the research period was set to 3 years, since this allowed for seasonal variability in disease prevalence.

\section{Results}

\section{Study population}

A total of 779 patients were admitted to the hospital with a primary diagnosis of acute ischemic stroke between January 1,2012 , and December 31, 2014 (Figure 1); 669 patients (288 lacunar infarctions, 141 atherothrombotic infarctions, 105 cardioembolic strokes, 23 specific-cause stroke, 102 insufficient minimum diagnostic assessments, 2 who were discharged dead, and 8 insufficient drug-criteria patients) were excluded. Thus, 110 ESUS patients were finally included in the study. Fifteen patients developed recurrent ischemic strokes over the 3-year follow-up: 14 of the 81 AP group patients and 1 of the 29 AC group patients. The 3-year follow up rate was 33.6\%. The mean follow-up period was 84.0 (standard deviation was 65.5) weeks.

\section{Patient characteristics}


The patients' demographics are shown in Table 1. The patients' median age at admission was 71.5 (SD, 13.2) years, and 31.8\% were women. The mRS score at discharge was 2.9 points in AC group patients and 2.4 points in AP group patients. Table 2 shows the antiplatelet drugs and doses used: 32 patients (39.5\%) used aspirin as single antiplatelet therapy; 25 used clopidogrel sulfate; and 20 used cilostazol. Three were administered double antiplatelet therapy. Among 29 AC group patients, three (10.3\%) used DOACs (two used apixaban 5 mg per day and one apixaban $10 \mathrm{mg}$ ). The warfarin control goal was an international normalized ratio (INR) of 2.0 - 3.0, but for patients over 70 years, it was 1.6 2.6 according to the Japanese guideline.[11] In the AC group, the recurrent stroke patient's INR was 2.54 at admission.

\section{Ischemic stroke recurrence rate of AC group patients}

The Kaplan-Meier recurrence-free survival rates are shown in Figure 2. Of 110 ESUS patients, 15 (13.6\%) developed ischemic stroke over the three years of follow-up, including one AC group patient (3.4\%) and 14 AP group patients (17.3\%).

\section{Multivariate analysis}

To clarify the significance of anticoagulant treatment as secondary prevention for ESUS, multivariate analysis of recurrence was performed using a Cox proportional hazard model; age, sex, $\mathrm{CHADS}_{2}$ score, history of smoking, diabetes mellitus, hypertension, dyslipidemia, stroke, history of chronic heart failure or acute coronary syndrome, and $\mathrm{mRS}$ score at discharge were not significantly associated with time to recurrence, whereas secondary prevention drug group (AP group or AC group) was a significant risk factor for recurrence (likelihood ratio -1.94, 95\% confidence interval $(\mathrm{Cl})$ : 0.01-0.72, $\mathrm{p}=0.013)$.

Table 3 shows recurrent stroke patients' characteristics. Their mean age was 77.9 years. Two of fifteen (13.3\%) were females, and only one patient received anticoagulant therapy. The mean recurrence-free period, eGFR, $\mathrm{CHADS}_{2}$ score, and mRS score were 64.5 weeks, 68.7

$\mathrm{mL} / \mathrm{min} / 1.73 \mathrm{~m}^{2}, 3.6$, and 2.5 , respectively. Twelve patients ( $\left.80 \%\right)$ had hypertension, and ten (66.7\%) were past or current smokers. No patients had acute coronary syndrome or heart failure.

\section{Overall survival and major bleeding}

Of 110 ESUS patients, 13 AP group patients and four AC group patients had died by three years. There was no overall survival difference between the AP group and AC group $(p=1,95 \% \mathrm{Cl}$ : 0.182-3.061) patients. No major bleeding meeting ISTH criteria occurred.

\section{Discussion}

The present results suggest that anticoagulants are more effective than antiplatelets for secondary prevention in ESUS patients. Antiplatelet drugs were recommended for ESUS as a result of the subgroup analysis of the Warfarin-Aspirin Recurrent Stroke Study (WARSS) trial.[5, 6] Recent studies showed that most cases of ESUS may be caused by cardioembolism,[7] but it has not been shown that anticoagulants are more effective for secondary prevention against ESUS than antiplatelets. In the present study, anticoagulants were more effective for secondary prevention than antiplatelets in such patients.

\section{Strength of this study:}

The main importance of this study is that an infarct was diagnosed using MRI in all patients. ESUS criteria included both MRI and CT images, but it is well known that MRI has greater sensitivity and specificity for acute ischemic stroke than CT.[16] By eliminating CT from the inclusion criteria, it was possible to diagnose acute ischemic stroke more definitively.

As a feature of this research, warfarin was prescribed to $89.7 \%$ of the anticoagulant-treated patients. Two global RCTs have already compared DOACs with antiplatelets, but there is no evidence that warfarin is more effective for preventing recurrent ischemic stroke than antiplatelets. There are still many situations when we need to use warfarin rather than DOACs, such as renal failure, cost issues, and so on. In the present study, whether there was a significant difference between warfarin and antiplatelets could not be determined, but this will be clarified in a future study.

This study showed the current regional Japanese status related to ESUS. Compared with the ESUS Global registry,[10] the ratios of ischemic stroke type and test insufficient were similar. Among the patients' characteristics, patients were older and had a higher recurrence rate than in the global registry, which is compatible with the Japanese background of these patients.

\section{Limitations of this study:}

The main limitation was patient selection. Since most patients did not undergo imaging of the extracranial arteries supplying the area of brain ischemia satisfying the ESUS criteria that were used, this was not set as an exclusion criterion. This differed from the ESUS criteria, but all patients underwent neck echo screening, and if the patient had severe neck artery stenosis, detailed examination was performed. 
Atherothrombotic infarctions were screened by other measures. This study was single-center cohort study, and the sample size was limited, but a good follow-up rate was expected because there were few other large hospitals in the area, and ischemic stroke patients were all seen at this hospital. To confirm the present findings, a multicenter, large sample size study is needed.

\section{Conclusions}

In summary, the present findings suggest that anticoagulants are more effective than antiplatelets for secondary stroke prevention for ESUS-like patients. Further studies are needed to accumulate more evidence.

\section{Abbreviations}

ESUS: Embolic stroke of undetermined source; DOACs: Direct oral anticoagulants; MRI: Magnetic resonance imaging; CT: Computed tomography; eGFR: Estimated glomerular filtration rate; AP: antiplatelet; AC: anticoagulant; mRS: modified Rankin Scale; TIAs: Transient ischemic attacks; ISTH: International Society on Thrombosis and Haemostasis; INR: International normalized ratio; CI: Confidence interval; HT: Hypertension; DM: Diabetes mellitus; DL: Dyslipidemia; HF: Heart failure; ACS: Acute coronary syndrome

\section{Declarations}

Ethics approval and consent to participate: The protocol was registered and approved by the Institutional Review Board of Seirei Hamamatsu General Hospital.

Consent for publication: Informed consent was not obtained as data were collected and reviewed retrospectively as part of a quality improvement, data were analyzed and presented in anonymized form, and there was no intervention. This approach was approved by the Institutional Review Board of Seirei Hamamatsu General Hospital.

\section{Availability of data and material:}

The datasets during and/or analyzed during the current study available from the corresponding author on reasonable request.

\section{Competing interests: None}

Funding: The authors declare that they have no competing interests.

Authors' Contributions: YS, YH and TT designed the study. YS and TA collected the patients' information. YS and TA contributed to collect clinical and laboratory data. Missing or abnormal data were checked by re-reviewing the medical charts by $\mathrm{YH}$. YS and YH analysis the data and wrote the manuscript. All authors read and approved the final paper.

\section{Acknowledgments}

The authors appreciate the contributions of Hiroshi Hori, MD, Takuya Watanabe, MD, PhD, Chikanori Inenaga MD, PhD, Yuki Amano, MD, Naoto Kuroda, MD, Yuichi Nakatogawa, MD, Daiki Uchida, MD, Mizuki Watanabe, MD, Tomohiro Yamazoe, MD, PhD, Hiroaki Kenmochi, MD, Toshihiko Ohashi, MD, Tsuyoshi Uchiyama, MD, PhD, Kenshiro Sato, MD, Daisuke Yamamoto, MD, Shigemasa Suzuki, MD, Kazuki Watanabe, MD, and Hironobu Myoujin, MD, who contributed to designing the study.

The authors would like to thank Forte Science Communications (https://www.fortescience.com/) for English language review.

\section{References}

1. Perera KS, Vanassche T, Bosch J, Swaminathan B, Mundl H, Giruparajah M, Barboza MA, O'Donnell MJ, Gomez-Schneider M, Hankey GJ et al: Global Survey of the Frequency of Atrial Fibrillation-Associated Stroke: Embolic Stroke of Undetermined Source Global Registry. Stroke 2016, 47(9):2197-2202.

2. Hart RG, Diener HC, Coutts SB, Easton JD, Granger CB, O'Donnell MJ, Sacco RL, Connolly SJ, Cryptogenic Stroke EIWG: Embolic strokes of undetermined source: the case for a new clinical construct. The Lancet Neurology 2014, 13(4):429-438.

3. Li L, Yiin GS, Geraghty OC, Schulz UG, Kuker W, Mehta Z, Rothwell PM, Oxford Vascular S: Incidence, outcome, risk factors, and long-term prognosis of cryptogenic transient ischaemic attack and ischaemic stroke: a population-based study. The Lancet Neurology 2015, 14(9):903-913.

4. Hart RG, Catanese L, Perera KS, Ntaios G, Connolly SJ: Embolic Stroke of Undetermined Source: A Systematic Review and Clinical Update. Stroke 2017, 48(4):867-872. 
5. Sacco RL, Prabhakaran S, Thompson JL, Murphy A, Sciacca RR, Levin B, Mohr JP, Investigators W: Comparison of warfarin versus aspirin for the prevention of recurrent stroke or death: subgroup analyses from the Warfarin-Aspirin Recurrent Stroke Study. Cerebrovascular diseases 2006, 22(1):4-12.

6. Mohr JP, Thompson JL, Lazar RM, Levin B, Sacco RL, Furie KL, Kistler JP, Albers GW, Pettigrew LC, Adams HP, Jr. et al: A comparison of warfarin and aspirin for the prevention of recurrent ischemic stroke. The New England journal of medicine 2001, 345(20):1444-1451.

7. Sposato LA, Cipriano LE, Saposnik G, Ruiz Vargas E, Riccio PM, Hachinski V: Diagnosis of atrial fibrillation after stroke and transient ischaemic attack: a systematic review and meta-analysis. The Lancet Neurology 2015, 14(4):377-387.

8. Hart RG, Sharma M, Mundl H, Kasner SE, Bangdiwala SI, Berkowitz SD, Swaminathan B, Lavados P, Wang Y, Wang Y et al: Rivaroxaban for Stroke Prevention after Embolic Stroke of Undetermined Source. The New England journal of medicine 2018, 378(23):2191-2201.

9. Diener HC, Easton JD, Granger CB, Cronin L, Duffy C, Cotton D, Brueckmann M, Sacco RL, Investigators R-SE: Design of Randomized, double-blind, Evaluation in secondary Stroke Prevention comparing the EfficaCy and safety of the oral Thrombin inhibitor dabigatran etexilate vs. acetylsalicylic acid in patients with Embolic Stroke of Undetermined Source (RE-SPECT ESUS). International journal of stroke : official journal of the International Stroke Society 2015, 10(8):1309-1312.

10. Perera KS, Vanassche T, Bosch J, Giruparajah M, Swaminathan B, Mattina KR, Berkowitz SD, Arauz A, O'Donnell MJ, Ameriso SF et al: Embolic strokes of undetermined source: Prevalence and patient features in the ESUS Global Registry. International journal of stroke : official journal of the International Stroke Society 2016, 11(5):526-533.

11. Ishihara H, Suzuki M: [Japanese Guidelines for the Management of Stroke 2015: overview of the chapter on Subarachnoid Hemorrhage]. Nihon rinsho Japanese journal of clinical medicine 2016, 74(4):677-680.

12. Wachter R, Groschel K, Gelbrich G, Hamann GF, Kermer P, Liman J, Seegers J, Wasser K, Schulte A, Jurries F et al: Holter-electrocardiogrammonitoring in patients with acute ischaemic stroke (Find-AFRANDOMISED): an open-label randomised controlled trial. The Lancet Neurology 2017, 16(4):282-290.

13. Gage BF, Waterman AD, Shannon W, Boechler M, Rich MW, Radford MJ: Validation of clinical classification schemes for predicting stroke: results from the National Registry of Atrial Fibrillation. Jama 2001, 285(22):2864-2870.

14. Schulman S, Kearon C, Subcommittee on Control of Anticoagulation of the S, Standardization Committee of the International Society on T, Haemostasis: Definition of major bleeding in clinical investigations of antihemostatic medicinal products in non-surgical patients. Journal of thrombosis and haemostasis : JTH2005, 3(4):692-694.

15. katano Takehiro KT, Mihara Ban, Kimura Kazumi: Embolic stroke of undetermined source区ESUS『. JStroke 2017, 39(6):470-475.

16. Chalela JA, Kidwell CS, Nentwich LM, Luby M, Butman JA, Demchuk AM, Hill MD, Patronas N, Latour L, Warach S: Magnetic resonance imaging and computed tomography in emergency assessment of patients with suspected acute stroke: a prospective comparison. Lancet 2007, 369(9558):293-298.

\section{Tables}

Table 1. Patients' demographics 


\begin{tabular}{|c|c|c|c|}
\hline & \multirow{2}{*}{$\begin{array}{c}\text { Total } \\
\mathrm{N}=110\end{array}$} & \multicolumn{2}{|c|}{ Treatment } \\
\hline & & $\begin{array}{l}\text { Anticoagulant } \\
\qquad(\mathrm{n}=29)\end{array}$ & $\begin{array}{c}\text { Antiplatelet } \\
(\mathrm{n}=81)\end{array}$ \\
\hline Mean age, y (SD) & $71.5( \pm 13.2)$ & 68.9 & 72.4 \\
\hline Female (\%) & 35 (31.8) & 18 & 24 \\
\hline Recurrent (\%) & $15(13.6)$ & $1(3.4)$ & $14(17.3)$ \\
\hline Mean weeks to recurrence, week (SD) & $84.0( \pm 65.5)$ & 21 & 67.6 \\
\hline \multicolumn{4}{|l|}{ Medical history } \\
\hline Hypertension (\%) & $58(52.7)$ & 13 & 45 \\
\hline Diabetes (\%) & $29(26.4)$ & 10 & 19 \\
\hline Hyperlipidemia (\%) & $47(42.7)$ & 13 & 34 \\
\hline Smoker (\%) & $45(40.9)$ & 12 & 33 \\
\hline Previous ischemic stroke (\%) & $35(31.8)$ & 10 & 25 \\
\hline Heart failure (\%) & $0(-)$ & 0 & 0 \\
\hline Acute coronary syndrome (\%) & $6(5.5)$ & 1 & 5 \\
\hline Mean eGFR, mL/min/1.73 m² (SD) & $70( \pm 24.2)$ & 68.5 & 70.5 \\
\hline Mean CHADS $_{2}$ score (SD) & $3.2( \pm 0.9)$ & 3.2 & 3.2 \\
\hline Mean mRS (SD) & $2.5( \pm 1.5)$ & 2.9 & 2.4 \\
\hline
\end{tabular}

SD, standard deviation; eGFR, estimated glomerular filtration rate; mRS score, modified Rankin Scale score at discharge.

Table 2. Antiplatelet agents and doses used

\begin{tabular}{ll}
\hline Number (person) & Drugs and doses (per day) \\
\hline 32 & Aspirin $100 \mathrm{mg}$ \\
20 & Clopidogrel sulfate $75 \mathrm{mg}$ \\
\hline 5 & Clopidogrel sulfate $25 \mathrm{mg}$ \\
\hline 16 & Cilostazol $200 \mathrm{mg}$ \\
\hline 4 & Cilostazol $100 \mathrm{mg}$ \\
\hline 2 & Aspirin $100 \mathrm{mg}$ and clopidogrel sulfate $75 \mathrm{mg}$ \\
\hline 1 & Aspirin $100 \mathrm{mg}$ and cilostazol $200 \mathrm{mg}$ \\
\hline 1 & Aspirin $100 \mathrm{mg}$ and cilostazol $100 \mathrm{mg}$ \\
\hline
\end{tabular}

Table 3. Recurrent stroke patients' characteristics 


\begin{tabular}{|c|c|c|c|c|c|c|c|c|c|c|c|c|c|}
\hline \multirow[t]{2}{*}{ Case } & \multirow{2}{*}{$\begin{array}{l}\text { Age } \\
\text { (Range) } \\
\text { (y) }\end{array}$} & \multirow[t]{2}{*}{ Treat } & \multirow{2}{*}{$\begin{array}{l}\text { Recurrence- } \\
\text { free } \\
\text { (weeks) }\end{array}$} & \multirow[b]{2}{*}{$\mathrm{HT}$} & \multirow[b]{2}{*}{$\mathrm{DM}$} & \multicolumn{3}{|c|}{ Medical History } & \multirow[b]{2}{*}{$\mathrm{HF}$} & \multirow[b]{2}{*}{ ACS } & \multirow{2}{*}{$\begin{array}{l}\text { eGFR } \\
(\mathrm{mL} / \mathrm{min} / 1.73 \\
\left.\mathrm{m}^{2}\right)\end{array}$} & \multirow[t]{2}{*}{$\mathrm{CHADS}_{2}$} & \multirow{2}{*}{$\begin{array}{l}\mathrm{mRS} \\
\text { score }\end{array}$} \\
\hline & & & & & & $\mathrm{DL}$ & Smoke & Stroke & & & & & \\
\hline 1 & $70-75$ & $\mathrm{AP}$ & 139 & + & - & - & - & - & - & - & 60 & 3 & 1 \\
\hline 2 & $75-80$ & $\mathrm{AC}$ & 21 & - & - & - & + & - & - & - & 76 & 3 & 3 \\
\hline 3 & $55-60$ & $\mathrm{AP}$ & 16 & - & - & + & - & - & - & - & 115 & 2 & 3 \\
\hline 4 & $80-85$ & $\mathrm{AP}$ & 23 & + & - & - & + & - & - & - & 83 & 4 & 2 \\
\hline 5 & $85-90$ & $\mathrm{AP}$ & 2 & + & - & - & + & + & - & - & 82 & 4 & 2 \\
\hline 6 & $85-90$ & $\mathrm{AP}$ & 82 & + & - & - & + & + & - & - & 73 & 4 & 2 \\
\hline 7 & $75-80$ & $\mathrm{AP}$ & 15 & + & + & + & + & - & - & - & 46 & 5 & 3 \\
\hline 8 & 81-85 & $\mathrm{AP}$ & 9 & + & - & + & + & - & - & - & 74 & 4 & 2 \\
\hline 9 & 80-85 & $\mathrm{AP}$ & 62 & + & - & + & - & + & - & - & 72 & 4 & 4 \\
\hline 10 & 80-85 & $\mathrm{AP}$ & 141 & - & - & + & - & - & - & - & 58 & 3 & 3 \\
\hline 11 & $70-75$ & $\mathrm{AP}$ & 21 & + & - & - & + & - & - & - & 71 & 3 & 4 \\
\hline 12 & $91-95$ & $\mathrm{AP}$ & 63 & + & + & - & + & + & - & - & 5 & 5 & 3 \\
\hline 13 & $65-70$ & $\mathrm{AP}$ & 125 & + & - & + & + & - & - & - & 82 & 3 & 4 \\
\hline 14 & $55-60$ & $\mathrm{AP}$ & 147 & + & - & - & + & - & - & - & 88 & 3 & 0 \\
\hline 15 & $85-90$ & $\mathrm{AP}$ & 102 & + & - & - & - & - & - & - & 45 & 4 & 2 \\
\hline
\end{tabular}

Treat indicates treatment drug; HT, hypertension; DM, diabetes mellitus; DL, dyslipidemia; HF, heart failure; ACS, acute coronary syndrome; eGFR, estimated glomerular filtration rate; mRS, modified Rankin Scale; AP, antiplatelet; AC, anticoagulant

Figures 
Figure 1.

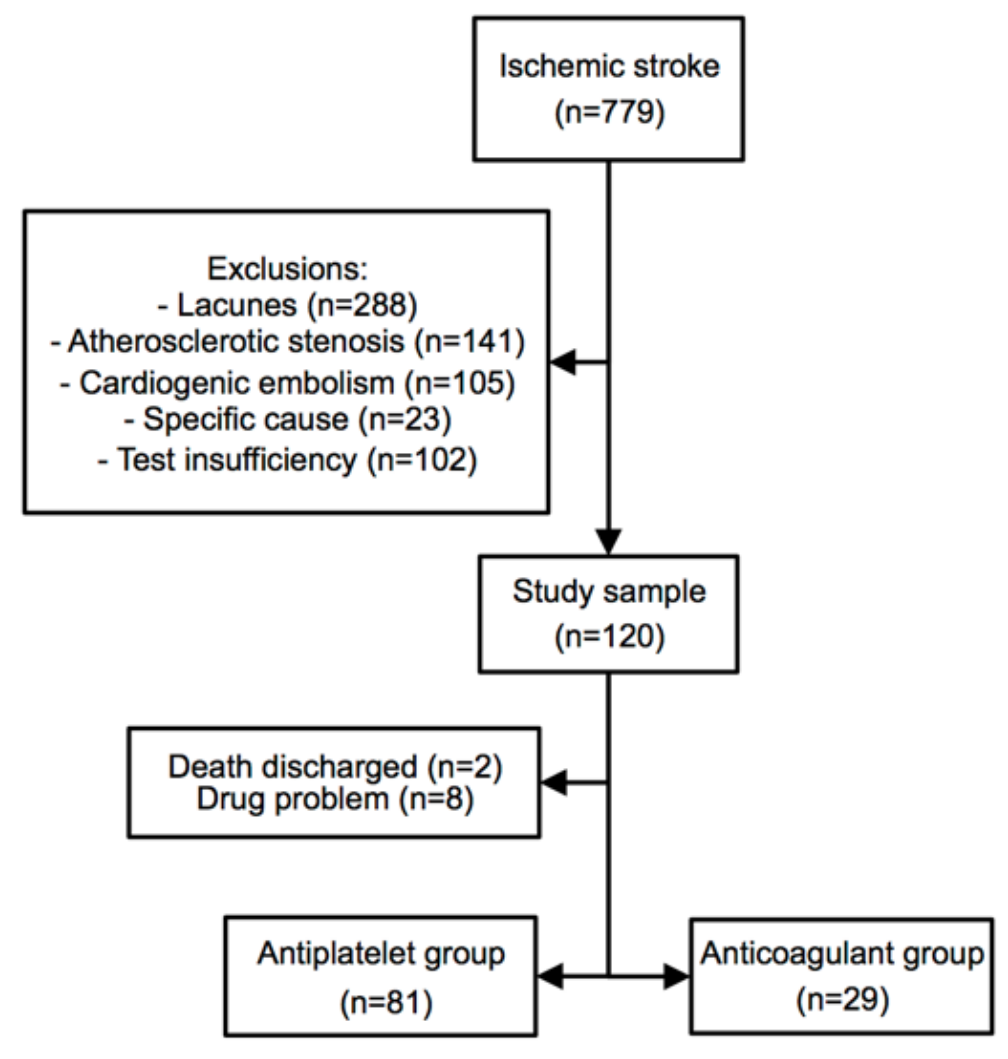

Figure 1

Flow chart of this study Ischemic stroke patients with positive neuroimaging were included, while those with cause known, those who were discharged dead, and those with insufficient drug criteria were excluded.

Figure 2.

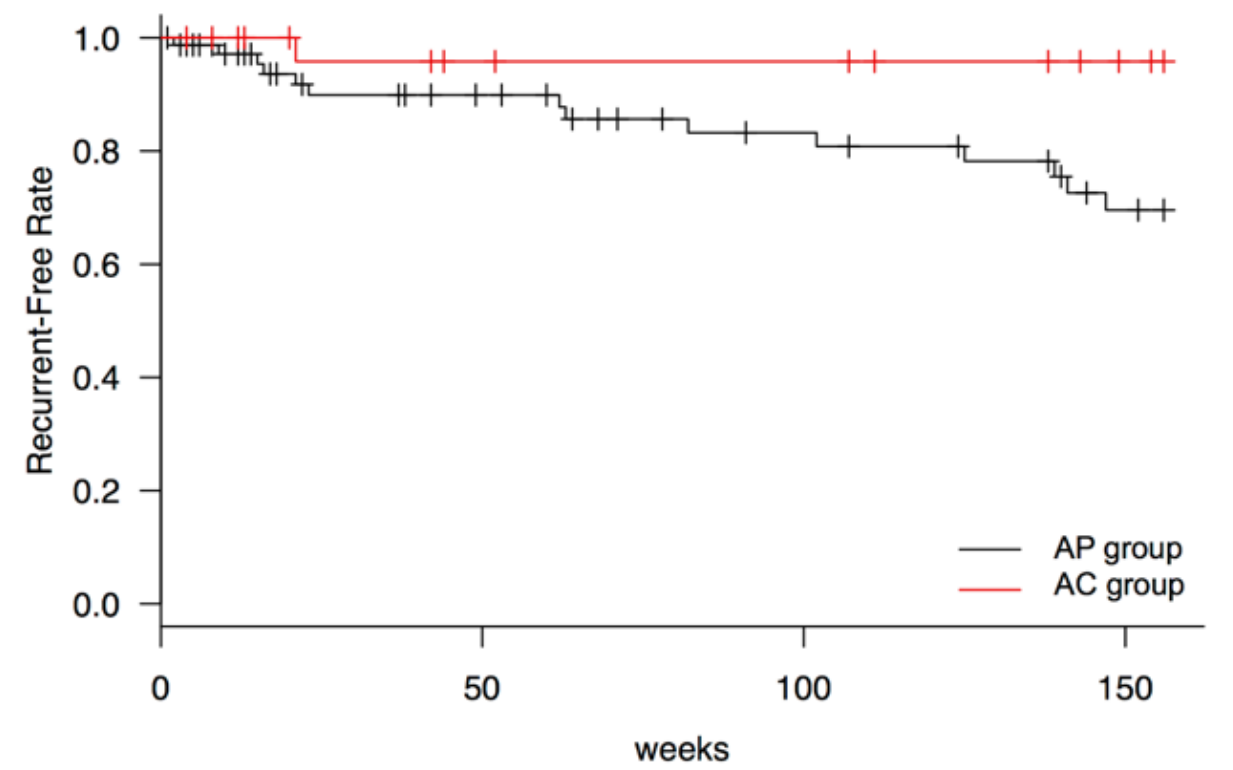

$\begin{array}{llll}\text { AP group } 81 & 44 & 34 & 23 \\ \text { AC group } 29 & 20 & 19 & 14\end{array}$


Figure 2

Kaplan-Meier survival analyses for recurrence in stroke patients with anticoagulants versus antiplatelets * Log-rank test $\mathrm{P}=0.0293$ ( $95 \% \mathrm{Cl}$ : 0.019-1.096). AP: antiplatelet, AC: anticoagulant 